\title{
Emergence and expansion of cosmic space in an accelerating BIon
}

\author{
Aroonkumar Beesham ${ }^{1, a}$, Alireza Sepehri ${ }^{1,2, b}$ \\ ${ }^{1}$ Department of Mathematical Sciences, University of Zululand, Private Bag X1001, Kwa-Dlangezwa, Richards Bay 3886, South Africa \\ ${ }^{2}$ Research Institute for Astronomy and Astrophysics of Maragha (RIAAM), P.O. Box 55134-441, Maragha, Iran
}

Received: 18 September 2018 / Accepted: 15 November 2018 / Published online: 23 November 2018

(C) The Author(s) 2018

\begin{abstract}
We generalize the Padmanabhan (arXiv:1206.4916 [hep-th]) mechanism to an accelerating BIon and show that the difference between the number of degrees of freedom on the boundary surface and the number of degrees of freedom in a bulk region causes the accelerated expansion of a BIon. We also consider the evolution of a universe which emerges on this BIon, and obtain its Hubble parameter and energy density.
\end{abstract}

\section{Introduction}

Several years ago, Padmanabhan suggested that the accelerated expansion of the universe is due to the difference between the surface degrees of freedom on the holographic horizon and the bulk degrees of freedom through the simple equation $\Delta V=\Delta t\left(N_{\text {sur }}-N_{\text {bulk }}\right)$, where $\mathrm{V}$ is the Hubble volume, and $t$ is the cosmic time, both expressed in Planck units [1]. Since then, many discussions have taken place on the Padmanabhan proposal [2-8]. For example, in one paper, with the help of this idea, the Friedmann equations of an $(n+1)$-dimensional Friedmann-Robertson-Walker universe corresponding to general relativity, Gauss-Bonnet gravity, and Lovelock gravity have been obtained [2]. In another, the idea of treating the cosmic space as an emergent process has been applied to brane cosmology, scalar-tensor cosmology, and $\mathrm{f}(\mathrm{R})$ gravity, and the corresponding cosmological equations in these theories have been derived [3]. In another investigation, using the Padmanabhan suggestion, the author obtained the Friedmann equations of the universe not only in four dimensional space-time and Einstein gravity, but also in higher dimensional space-time and other gravity theories like Gauss-Bonnet and Lovelock gravity with any spacial curvature [4].

Some other authors, have extended the evolution equation in the Padmanabhan idea to give the Friedmann equation

\footnotetext{
a e-mail: beeshama@unizulu.ac.za

be-mail: alireza.sepehri3@gmail.com
}

in the nonflat universe corresponding to $k= \pm 1$ by taking into account the invariant volume surrounded by the apparent horizon [5]. In another scenario, the authors showed that applying Padmanabhan's conjecture to non-Einstein gravity cases encounters serious difficulties and has to be heavily modified to get the Friedmann equation [6]. In another paper, Ali applied the equations of the universe derived in the Padmanabhan model with the corrected entropy-area law that follows from the Generalized Uncertainty Principle (GUP) and obtained a modified Friedmann equation due to the GUP [7]. In more recent research, the Padmanabhan idea has been constructed in a BIonic system and it was shown that all degrees of freedom inside and outside the universe are controlled by the evolution of the BIon in the extra dimensions and tend to degrees of freedom of the black F-string in string theory [8].

The main question that arises is what is the origin of this inequality between the surface degrees of freedom and the bulk degrees of freedom? We answer this question in an accelerating BIon. A BIon is a configuration which has been constructed from a brane, an anti-brane and a wormhole which connects them. In our investigation, branes are expanding with acceleration. We show that the acceleration of the branes in this BIon leads to the difference between the number of the degrees of freedom on the surface of BIon and that in a bulk.

The outline of the paper is as follows. In Sect. 2, we will obtain numbers of degrees of freedom on the surface and bulk. In Sect. 3, we will consider the evolution of a universe which emerges on the branes of this accelerating BIon.

\section{Padmanabhan mechanism in an accelerating BIon}

In this section, we will consider the Padmanabhan mechanism in an accelerating BIon. We will show that the acceleration of the BIon leads to the difference between the number of degrees of freedom on the surface and the number in a bulk. 


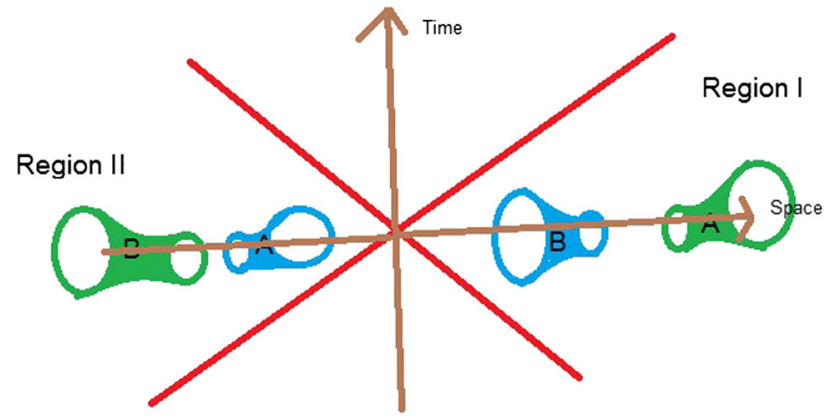

Fig. 1 Two parts of BIons in two regions of the Rindle space-time. Parts with green color are expanding and parts with blue color are contracting

Previously, it has been shown that the metric of a thermal BIon in 10-dimensional space-time is given by $[9,10]$

$$
\begin{aligned}
d s^{2}= & D^{-\frac{1}{2}} H^{-\frac{1}{2}}\left(d x_{2}^{2}+d x_{3}^{2}\right) \\
& +D^{\frac{1}{2}} H^{-\frac{1}{2}}\left(-f d t^{2}+d x_{1}^{2}\right) \\
& +D^{-\frac{1}{2}} H^{\frac{1}{2}}\left(f^{-1} d r^{2}+r^{2} d \Omega_{5}^{2}\right)
\end{aligned}
$$

where

$$
\begin{aligned}
& f=1-\frac{r_{0}^{4}}{r^{4}} \quad H=1+\frac{r_{0}^{4} \sinh ^{2} \alpha}{r^{4}} \\
& D=\cos ^{2} \epsilon+\sin ^{2} \epsilon H^{-1}
\end{aligned}
$$

and

$$
\cosh ^{2} \alpha=\frac{3}{2} \frac{\cos \frac{\delta}{3}+\sqrt{3} \cos \frac{\delta}{3}}{\cos \delta} \quad \cos \epsilon=\frac{1}{\sqrt{1+\frac{K^{2}}{r^{4}}}}
$$

The angle $\delta$ is defined as:

$\cos \delta=\bar{T}^{4} \sqrt{1+\frac{K^{2}}{r^{4}}} \bar{T}=\left(\frac{9 \pi^{2} N}{4 \sqrt{3} T_{D 3}}\right)^{\frac{1}{2}} T$

In an accelerating BIon, the relation between the world volume of the coordinates of the accelerating D3-branes $(\tau, \sigma)$ and the coordinates of 10D Minkowski space-time $(t, r)$ are [11];

$$
\begin{aligned}
& a t=e^{a \sigma} \sinh (a \tau) \quad a r=e^{a \sigma} \cosh (a \tau) \quad \text { In Region I } \\
& a t=-e^{-a \sigma} \sinh (a \tau) \quad a r=e^{-a \sigma} \cosh (a \tau) \quad \text { In Region II }
\end{aligned}
$$

where $a$ is the acceleration of the branes. The above equation shows that the acceleration leads to the emergence of a Rindler space-time. This space-time has two regions and the BIons in each region act in reverse to the BIons in the other region. Also, each BIon has two parts (A and $\mathrm{B}$ ). When the branes in part A expand, the branes in part $\mathrm{B}$ contract, and vice versa (see Fig. 1).

Substituting the results of Eq. (5) into Eq. (1), we obtain [12]:

$$
\begin{aligned}
d s_{I, A, \text { thermal }}^{2} & =D_{I-A}^{\frac{1}{2}} H_{I-A}^{-\frac{1}{2}} f_{I-A}\left(e^{2 a \sigma}+\frac{1}{\sinh ^{2}(a \tau)}\left(\frac{d z}{d \tau}\right)^{2}\right) d \tau^{2} \\
& -D_{I-A}^{-\frac{1}{2}} H_{I-A}^{\frac{1}{2}} f_{I-A}^{-1}\left(e^{2 a \sigma}+\frac{1}{\cosh ^{2}(a \tau)}\left(\frac{d z}{d \sigma}\right)^{2}\right) d \sigma^{2} \\
& +\frac{1}{\sinh (a \tau) \cosh (a \tau)}\left(\frac{d z}{d \tau} \frac{d z}{d \sigma}\right) d \tau d \sigma \\
& +D_{I-A}^{-\frac{1}{2}} H_{I-A}^{\frac{1}{2}}\left(\frac{1}{a} e^{a \sigma} \cosh (a \tau)\right)^{2}\left(d \theta^{2}+\sin ^{2} \theta d \phi^{2}\right) \\
& +D_{I-A}^{-\frac{1}{2}} H_{I-A}^{-\frac{1}{2}} \sum_{i=1}^{5} d x_{i}^{2} \\
d s_{I I, A, t h e r m a l}^{2} & =D_{I I-A}^{\frac{1}{2}} H_{I I-A}^{-\frac{1}{2}} f_{I I-A} \\
& \times\left(e^{-2 a \sigma}+\frac{1}{\sinh ^{2}(a \tau)}\left(\frac{d z}{d \tau}\right)^{2}\right) d \tau^{2} \\
& -D_{I I-A}^{-\frac{1}{2}} H_{I I-A}^{\frac{1}{2}} f_{I I-A}^{-1} \\
& \times\left(e^{-2 a \sigma}+\frac{1}{\cosh ^{2}(a \tau)}\left(\frac{d z}{d \sigma}\right)^{2}\right) d \sigma^{2} \\
& -\frac{1}{\sinh (a \tau) \cosh ^{2}(a \tau)}\left(\frac{d z}{d \tau} \frac{d z}{d \sigma}\right) d \tau d \sigma \\
& +D_{I I-A}^{-\frac{1}{2}} H_{I I-A}^{\frac{1}{2}}\left(\frac{1}{a} e^{-a \sigma} \cosh (a \tau)\right)^{2} \times\left(d \theta^{2}+\sin ^{2} \theta d \phi^{2}\right) \\
& +D_{I I-A}^{-\frac{1}{2}} H_{I I-A}^{-\frac{1}{2}} \sum_{i=1}^{5} d x_{i}^{2}
\end{aligned}
$$

where

$$
\begin{aligned}
f_{I-A} & =1-\frac{\left(e^{a \sigma_{0}} \cosh \left(a \tau_{0}\right)\right)^{4}}{\left(e^{a \sigma} \cosh (a \tau)\right)^{4}} H_{I-A} \\
& =1+\frac{\left(e^{a \sigma_{0}} \cosh \left(a \tau_{0}\right)\right)^{4} \sinh ^{2} \alpha_{I-A}}{\left(e^{a \sigma} \cosh (a \tau)\right)^{4}} \\
D_{I-A} & =\cos ^{2} \epsilon_{I-A}+\sin ^{2} \epsilon_{I-A} H_{I-A}^{-1} \\
f_{I I-A} & =1-\frac{\left(e^{-a \sigma_{0}} \cosh \left(a \tau_{0}\right)\right)^{4}}{\left(e^{-a \sigma} \cosh (a \tau)\right)^{4}} H_{I I-A} \\
& =1+\frac{\left(e^{a \sigma_{0}} \cosh \left(a \tau_{0}\right)\right)^{4} \sinh ^{2} \alpha_{I I-A}}{\left(e^{a \sigma} \cosh (a \tau)\right)^{4}} \\
D_{I I-A} & =\cos ^{2} \epsilon_{I I-A}+\sin ^{2} \epsilon_{I I-A} H_{I I-A}^{-1}
\end{aligned}
$$

and

$$
\begin{aligned}
\cosh ^{2} \alpha_{I-A} & =\frac{3}{2} \frac{\cos \frac{\delta_{I-A}}{3}+\sqrt{3} \cos \frac{\delta_{I-A}}{3}}{\cos \delta_{I-A}} \\
\cos \epsilon_{I-A} & =\frac{1}{\sqrt{1+\frac{K^{2}}{\left(a^{-1} e^{-a \sigma} \cosh (a \tau)\right)^{4}}}} \\
\cosh ^{2} \alpha_{I I-A} & =\frac{3}{2} \frac{\cos \frac{\delta_{I I-A}}{3}+\sqrt{3} \cos \frac{\delta_{I I-A}}{3}}{\cos \delta_{I I-A}}
\end{aligned}
$$




$$
\cos \epsilon_{I I-A}=\frac{1}{\sqrt{1+\frac{K^{2}}{\left(a^{-1} e^{a \sigma} \cosh (a \tau)\right)^{4}}}}
$$

The angles $\delta_{I-A}$ and $\delta_{I I-A}$ are defined by:

$$
\begin{aligned}
\cos \delta_{I-A} & =\bar{T}_{0, I-A}^{4} \sqrt{1+\frac{K^{2}}{\left(a^{-1} e^{-a \sigma} \cosh (a \tau)\right)^{4}}} \\
\bar{T}_{0, I-A} & =\left(\frac{9 \pi^{2} N}{4 \sqrt{3} T_{D 3}}\right)^{\frac{1}{2}} T_{0, I-A} \\
\cos \delta_{I I-A} & =\bar{T}_{0, I I-A}^{4} \sqrt{1+\frac{K^{2}}{\left(a^{-1} e^{a \sigma} \cosh (a \tau)\right)^{4}}} \\
\bar{T}_{0, I I-A} & =\left(\frac{9 \pi^{2} N}{4 \sqrt{3} T_{D 3}}\right)^{\frac{1}{2}} T_{0, I I-A}
\end{aligned}
$$

where $T_{0}$ is the temperature of the BIon in non-Rindler spacetime. For the above metric, the energy densities and entropies in the two regions are:

$$
\begin{aligned}
\int d \sigma \frac{d M_{I-A}}{d z}= & \int d \sigma \frac{d M_{I I-B}}{d z}=\frac{4 T_{D 3}^{2}}{\pi T_{0, I-A}^{4}} \int_{\sigma_{0}}^{\infty} d \sigma \frac{F_{D B I, I, A}(\sigma, \tau)\left(\frac{1}{a} e^{a \sigma} \cosh (a \tau)\right)^{2}\left(\sinh ^{2}(a \tau)+\cosh ^{2}(a \tau)\right)}{\sqrt{F_{D B I, I, A}^{2}(\sigma, \tau)-F_{D B I, I, A}^{2}\left(\sigma_{o}, \tau\right)}} \\
& \times \frac{4 \cosh ^{2} \alpha_{I-A}+1}{\cosh ^{4} \alpha_{I-A}} \\
S_{I-A}=S_{I I-B}= & \frac{4 T_{D 3}^{2}}{\pi T_{0, I-A}^{5}} \int_{\sigma_{0}}^{\infty} d \sigma \frac{F_{D B I, I, A}(\sigma, \tau)\left(\frac{1}{a} e^{a \sigma} \cosh (a \tau)\right)^{2}\left(\sinh ^{2}(a \tau)+\cosh ^{2}(a \tau)\right)}{\sqrt{F_{D B I, I, A}^{2}(\sigma, \tau)-F_{D B I, I, A}^{2}\left(\sigma_{o}, \tau\right)}} \\
& \times \frac{4}{\cosh ^{4} \alpha_{I-A}}
\end{aligned}
$$

with the definition of $F_{D B I, I, A}$ given below:

$$
\begin{aligned}
F_{D B I, I, A}= & F_{D B I, I I, B}=\left(a^{-1} e^{a \sigma} \cosh (a \tau)\right)^{2} \\
& \times \frac{4 \cosh ^{2} \alpha_{I-A}-3}{\cosh ^{4} \alpha_{I-A}}
\end{aligned}
$$

and

$$
\begin{aligned}
\int d \sigma \frac{d M_{I-B}}{d z}= & \int d \sigma \frac{d M_{I I-A}}{d z}=\frac{4 T_{D 3}^{2}}{\pi T_{0, I I-A}^{4}} \int_{\sigma_{0}}^{\infty} d \sigma \frac{F_{D B I, I I, A}(\sigma, \tau)\left(\frac{1}{a} e^{-a \sigma} \cosh (a \tau)\right)^{2}\left(\sinh ^{2}(a \tau)+\cosh ^{2}(a \tau)\right)}{\sqrt{F_{D B I, I I, A}^{2}(\sigma, \tau)-F_{D B I, I I, A}^{2}\left(\sigma_{o}, \tau\right)}} \\
& \times \frac{4 \cosh ^{2} \alpha_{I I-A}+1}{\cosh ^{4} \alpha_{I I-A}} \\
S_{I I-A}=S_{I-B}= & \frac{4 T_{D 3}^{2}}{\pi T_{0, I I-A}^{5}} \int_{\sigma_{0}}^{\infty} d \sigma \frac{F_{D B I, I I, A}(\sigma, \tau)\left(\frac{1}{a} e^{-a \sigma} \cosh (a \tau)\right)^{2}\left(\sinh ^{2}(a \tau)+\cosh ^{2}(a \tau)\right)}{\sqrt{F_{D B I, I I, A}^{2}(\sigma, \tau)-F_{D B I, I I, A}^{2}\left(\sigma_{o}, \tau\right)}} \\
& \times \frac{4}{\cosh ^{4} \alpha_{I I-A}}
\end{aligned}
$$

$$
\begin{aligned}
& N_{s u r, I-A}-N_{b u l k, I-A}=\int d \sigma \frac{d M_{I-A}}{d z}=\int d \sigma \frac{d M_{I I-B}}{d z} \\
& N_{\text {sur }, I-A}+N_{\text {bulk,I-A }}=\frac{4 \pi}{L_{P}^{2}} S_{I-A}=\frac{4 \pi}{L_{P}^{2}} S_{I I-B} \\
& N_{s u r, I-B}-N_{b u l k, I-B}=\int d \sigma \frac{d M_{I-B}}{d z} \\
& =\int d \sigma \frac{d M_{I I-A}}{d z} \\
& N_{\text {sur }, I-B}+N_{\text {bulk, } I-B}=\frac{4 \pi}{L_{P}^{2}} S_{I-B}=\frac{4 \pi}{L_{P}^{2}} S_{I I-A} \\
& F_{D B I, I I, A}=F_{D B I, I, B}=\left(a^{-1} e^{-a \sigma} \cosh (a \tau)\right)^{2} \\
& \frac{4 \cosh ^{2} \alpha_{I I-A}-3}{\cosh ^{4} \alpha_{I I-A}}
\end{aligned}
$$

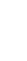


Consequently, for $L_{P}^{2}=4 \pi$, the numbers of degrees of freedom on the surface and in the bulk are: where $T$ is temperature of the BIon and $T_{0}$ is the critical temperature relating to the colliding point of the branes.

$$
\begin{aligned}
N_{\text {sur }, I-A}= & \frac{4 T_{D 3}^{2}}{\pi T_{0, I-A}^{4}} \int_{\sigma_{0}}^{\infty} d \sigma \frac{F_{D B I, I, A}(\sigma, \tau)\left(\frac{1}{a} e^{a \sigma} \cosh (a \tau)\right)^{2}\left(\sinh ^{2}(a \tau)+\cosh ^{2}(a \tau)\right)}{\sqrt{F_{D B I, I, A}^{2}(\sigma, \tau)-F_{D B I, I, A}^{2}\left(\sigma_{o}, \tau\right)}} \\
& \times \frac{4 \cosh ^{2} \alpha_{I-A}+5}{\cosh ^{4} \alpha_{I-A}} \\
N_{\text {bulk }, I-A}= & \frac{4 T_{D 3}^{2}}{\pi T_{0, I-A}^{4}} \int_{\sigma_{0}}^{\infty} d \sigma \frac{F_{D B I, I, A}(\sigma, \tau)\left(\frac{1}{a} e^{a \sigma} \cosh (a \tau)\right)^{2}\left(\sinh ^{2}(a \tau)+\cosh ^{2}(a \tau)\right)}{\sqrt{F_{D B I, I, A}^{2}(\sigma, \tau)-F_{D B I, I, A}^{2}\left(\sigma_{o}, \tau\right)}} \\
& \times \frac{4 \cosh ^{2} \alpha_{I-A}-3}{\cosh ^{4} \alpha_{I-A}} \\
N_{\text {sur }, I-B}= & \int d \sigma \frac{d M_{I I-A}}{d z}=\frac{4 T_{D 3}^{2}}{\pi T_{0, I I-A}^{4}} \int_{\sigma_{0}}^{\infty} d \sigma \frac{F_{D B I, I I, A}(\sigma, \tau)\left(\frac{1}{a} e^{-a \sigma} \cosh (a \tau)\right)^{2}\left(\sinh ^{2}(a \tau)+\cosh ^{2}(a \tau)\right)}{\sqrt{F_{D B I, I I, A}^{2}(\sigma, \tau)-F_{D B I, I I, A}^{2}\left(\sigma_{o}, \tau\right)}} \\
& \times \frac{4 \cosh ^{2} \alpha_{I I-A}+5}{\cosh ^{4} \alpha_{I I-A}} \\
N_{\text {bulk,I-B}=}= & \int d \sigma \frac{d M_{I I-A}}{d z}=\frac{4 T_{D 3}^{2}}{\pi T_{0, I I-A}^{4}} \int_{\sigma_{0}}^{\infty} d \sigma \frac{F_{D B I, I I, A}(\sigma, \tau)\left(\frac{1}{a} e^{-a \sigma} \cosh (a \tau)\right)^{2}\left(\sinh ^{2}(a \tau)+\cosh ^{2}(a \tau)\right)}{\sqrt{F_{D B I, I I, A}^{2}(\sigma, \tau)-F_{D B I, I I, A}^{2}\left(\sigma_{o}, \tau\right)}} \\
& \times \frac{4 \cosh ^{2} \alpha_{I I-A}-3}{\cosh ^{4} \alpha_{I I-A}}
\end{aligned}
$$

The above equations show that the number of degrees of freedom depends on the acceleration of the branes and the parameters of the BIon in the extra dimensions. By increasing the acceleration, the number of degrees of freedom on the surface of one of parts increases, and number of degrees of freedom in the bulk for it correspondingly decreases, while, the number of degrees of freedom on the surface of another part decreases and number of degrees of freedom on its bulk correspondingly increases.

\section{Evolution of the universe in an accelerating BIon}

In this section, we will consider the evolution of a universe which emerges in an accelerating BIon. To this aim, we will obtain the number of degrees of freedom in terms of temperature.

Until now, various relations for temperature of a moving system have been proposed. For example:

$$
\begin{aligned}
\begin{array}{l}
v \\
=
\end{array} & a \tau \\
& \rightarrow T=\frac{T_{0}}{\sqrt{1-\frac{v^{2}}{c^{2}}}}=\frac{T_{0}}{\sqrt{1-\frac{[a \tau]^{2}}{c^{2}}}} \\
v & =a \tau=c \sqrt{1-\frac{T_{0}^{2}}{T^{2}}}
\end{aligned}
$$

However this relation is questionable. Based on this relation, the superconductivity phenomena depends on the system velocity!! You can move a system with special velocities to reduce its temperature to less than that of its critical temperature, and then the system shows superconductivity by itself!! In fact, it means that a physical phenomena (superconductivity) depends on the system velocity, a result in direct conflict with the relativity law claiming that physical laws are independent of the observer velocity. This relativistic relation for temperature is not a true relation, and in fact, the temperature's relation depends on the thermocouple apparatus used. A true thermocouple rejects this definition of temperature (For example, see [1315]). Thus, to obtain the true relation between temperature and acceleration, we make use of the concepts of the BIon:

$$
d M_{I-A / B}=T_{I-A / B} d S_{I-A / B} \rightarrow T_{I-A / B}=\frac{d M_{I-A / B}}{d S_{I-A / B}}
$$

Previously, thermodynamical parameters have been obtained in [12]: 


$$
\begin{aligned}
& d M_{I-A}=\frac{d M_{I-A}}{d z_{I-A}} d z_{I-A} \\
& d z_{I-A}=d z_{I I-B} \simeq\left(e^{-4 a \sigma} \sinh ^{2}(a \tau) \cosh ^{2}(a \tau)\right)
\end{aligned}
$$

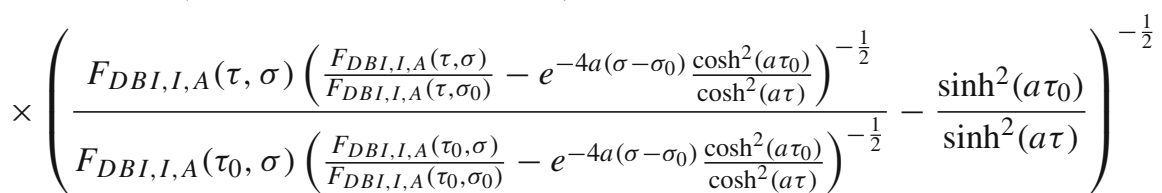

$$
\begin{aligned}
& \frac{d M_{I-A}}{d z}=\frac{d M_{I I-B}}{d z}=\frac{4 T_{D 3}^{2}}{\pi T_{0, I-A}^{4}} \frac{F_{D B I, I, A}(\sigma, \tau)\left(\frac{1}{a} e^{a \sigma} \cosh (a \tau)\right)^{2}\left(\sinh ^{2}(a \tau)+\cosh ^{2}(a \tau)\right)}{\sqrt{F_{D B I, I, A}^{2}(\sigma, \tau)-F_{D B I, I, A}^{2}\left(\sigma_{o}, \tau\right)}} \\
& \times \frac{4 \cosh ^{2} \alpha_{I-A}+1}{\cosh ^{4} \alpha_{I-A}} \\
& d S_{I-A}=d S_{I I-B}=\frac{4 T_{D 3}^{2}}{\pi T_{0, I-A}^{5}} \frac{F_{D B I, I, A}(\sigma, \tau)\left(\frac{1}{a} e^{a \sigma} \cosh (a \tau)\right)^{2}\left(\sinh ^{2}(a \tau)+\cosh ^{2}(a \tau)\right)}{\sqrt{F_{D B I, I, A}^{2}(\sigma, \tau)-F_{D B I, I, A}^{2}\left(\sigma_{o}, \tau\right)}} \\
& \times \frac{4}{\cosh ^{4} \alpha_{I-A}}
\end{aligned}
$$

and

$$
\begin{aligned}
& d M_{I-B}=\frac{d M_{I-B}}{d z_{I-B}} d z_{I-B} \\
& d z_{I-B}=d z_{I I-A} \simeq\left(e^{4 a \sigma} \sinh ^{2}(a \tau) \cosh ^{2}(a \tau)\right)
\end{aligned}
$$

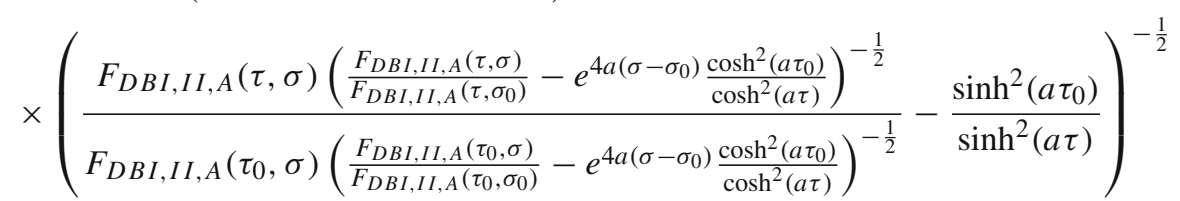

$$
\begin{aligned}
& \frac{d M_{I-B}}{d z}=\frac{d M_{I I-A}}{d z}=\frac{4 T_{D 3}^{2}}{\pi T_{0, I I-A}^{4}} \frac{F_{D B I, I I, A}(\sigma, \tau)\left(\frac{1}{a} e^{-a \sigma} \cosh (a \tau)\right)^{2}\left(\sinh ^{2}(a \tau)+\cosh ^{2}(a \tau)\right)}{\sqrt{F_{D B I, I I, A}^{2}(\sigma, \tau)-F_{D B I, I I, A}^{2}\left(\sigma_{o}, \tau\right)}} \\
& \times \frac{4 \cosh ^{2} \alpha_{I I-A}+1}{\cosh ^{4} \alpha_{I I-A}} \\
& d S_{I I-A}=d S_{I-B}=\frac{4 T_{D 3}^{2}}{\pi T_{0, I I-A}^{5}} \frac{F_{D B I, I I, A}(\sigma, \tau)\left(\frac{1}{a} e^{-a \sigma} \cosh (a \tau)\right)^{2}\left(\sinh ^{2}(a \tau)+\cosh ^{2}(a \tau)\right)}{\sqrt{F_{D B I, I I, A}^{2}(\sigma, \tau)-F_{D B I, I I, A}^{2}\left(\sigma_{o}, \tau\right)}} \\
& \times \frac{4}{\cosh ^{4} \alpha_{I I-A}}
\end{aligned}
$$

Using relation (24 and 25) in relation (23), we can obtain the explicit form of the temperature in an accelerating BIon as: 


$$
\begin{aligned}
T_{I-A}= & T_{0, I-A}\left(4 \cosh ^{2} \alpha_{I-A}+1\right) \times\left(e^{-4 a \sigma} \sinh ^{2}(a \tau) \cosh ^{2}(a \tau)\right) \\
& \times\left(\frac{F_{D B I, I, A}(\tau, \sigma)\left(\frac{F_{D B I, I, A}(\tau, \sigma)}{F_{D B I, I, A}\left(\tau, \sigma_{0}\right)}-e^{-4 a\left(\sigma-\sigma_{0}\right)} \frac{\cosh ^{2}\left(a \tau_{0}\right)}{\cosh ^{2}(a \tau)}\right)^{-\frac{1}{2}}}{F_{D B I, I, A}\left(\tau_{0}, \sigma\right)\left(\frac{F_{D B I, I, A}\left(\tau_{0}, \sigma\right)}{F_{D B I, I, A}\left(\tau_{0}, \sigma_{0}\right)}-e^{-4 a\left(\sigma-\sigma_{0}\right)} \frac{\cosh ^{2}\left(a \tau_{0}\right)}{\cosh ^{2}(a \tau)}\right)^{-\frac{1}{2}}}-\frac{\sinh ^{2}\left(a \tau_{0}\right)}{\sinh ^{2}(a \tau)}\right)^{\frac{1}{2}}
\end{aligned}
$$

and

$$
\begin{aligned}
T_{I-B}= & T_{0, I-B}\left(4 \cosh ^{2} \alpha_{I-B}+1\right) \times\left(e^{4 a \sigma} \sinh ^{2}(a \tau) \cosh ^{2}(a \tau)\right) \\
& \times\left(\frac{F_{D B I, I, B}(\tau, \sigma)\left(\frac{F_{D B I, I, B}(\tau, \sigma)}{F_{D B I, I, B}\left(\tau, \sigma_{0}\right)}-e^{4 a\left(\sigma-\sigma_{0}\right)} \frac{\cosh ^{2}\left(a \tau_{0}\right)}{\cosh ^{2}(a \tau)}\right)^{-\frac{1}{2}}}{F_{D B I, I, B}\left(\tau_{0}, \sigma\right)\left(\frac{F_{D B I, I, B}\left(\tau_{0}, \sigma\right)}{F_{D B I, I, B}\left(\tau_{0}, \sigma_{0}\right)}-e^{4 a\left(\sigma-\sigma_{0}\right)} \frac{\cosh ^{2}\left(a \tau_{0}\right)}{\cosh ^{2}(a \tau)}\right)^{-\frac{1}{2}}}-\frac{\sinh ^{2}\left(a \tau_{0}\right)}{\sinh ^{2}(a \tau)}\right)^{\frac{1}{2}}
\end{aligned}
$$

On the other hand, previously, it has been shown that the Hubble parameter of the universe has the following relation with the number of degrees of freedom on the surface of the BIon [8]:

$$
\begin{aligned}
N_{\text {sur }} & =\frac{4 \pi r_{A}^{2}}{L_{P}^{2}} \\
r_{A} & =\sqrt{H^{2}+\frac{k^{2}}{\bar{a}^{2}}}
\end{aligned}
$$

where $H=\frac{\bar{a}}{\bar{a}}$ is the Hubble parameter, $\bar{a}$ is the scale factor and $r_{A}$ is the apparent horizon radius for the FRW universe. Using Eqs. (20, 21, 22, 23, 24, 25, 26, 27 and 28), we can obtain the Hubble parameter and scale factor of the flat universe $(k=0)$ on each part of the BIon:

$$
\begin{aligned}
& H_{I-A} \simeq \frac{313 \pi^{14} k^{14} c^{14} L_{P}^{2}}{T_{D 3}^{8} \sigma_{0}^{73}} \\
& \times e^{\left[1-\left[1-\frac{\tau^{2}}{\tau_{0}^{2}}\right]^{1 / 2} \frac{T_{0}^{2}}{T^{2}}\right]^{8}\left[1-\left[1-\frac{\sigma^{2}}{\sigma_{0}^{2}}\right]^{1 / 2} \frac{T_{0}^{2}}{T^{2}}\right]^{5}} \\
& \times\left[1-\left[1-\frac{\tau^{2}}{\tau_{0}^{2}}\right]^{1 / 2} \frac{T_{0}^{2}}{T^{2}}\right]^{73} \\
& \times\left[1+\frac{8 \pi^{5} k^{5} c^{5} L_{P}^{2}}{T_{D 3}^{313} \sigma_{0}^{313}}\left[1-\left[1-\frac{\sigma^{2}}{\sigma_{0}^{2}}\right]^{1 / 2} \frac{T_{0}^{2}}{T^{2}}\right]^{313}\right] \\
& \bar{a}_{I-A}=e^{\int d t H_{I-A}} \\
& H_{I-B} \simeq \frac{313 \pi^{14} k^{14} c^{14} L_{P}^{2}}{T_{D 3}^{8} \sigma_{0}^{73}} \\
& \quad \times e^{-\left[1-\left[1-\frac{\tau^{2}}{\tau_{0}^{2}}\right]^{1 / 2} \frac{T_{0}^{2}}{T^{2}}\right]^{8}\left[1-\left[1-\frac{\sigma^{2}}{\sigma_{0}^{2}}\right]^{1 / 2} \frac{T_{0}^{2}}{T^{2}}\right]^{5}}
\end{aligned}
$$

$$
\times \frac{1}{\left[1-\left[1-\frac{\tau^{2}}{\tau_{0}^{2}}\right]^{1 / 2} \frac{T_{0}^{2}}{T^{2}}\right]^{73}}
$$

$\bar{a}_{I-A}=e^{\int d t H_{I-A}}$

The above equations show that by increasing the temperature of the BIon and the acceleration of system, the Hubble parameter and scale factor of the universe which lives on part A of the BIon in region I decrease, while the Hubble parameter and scale factor of the universe on part B of the BIon in region I increase. This means that by expanding one universe, another universe contracts.

On the other hand, using the Friedmann equation of the flat FRW universe, we can calculate the energy density of the universe:

$$
\begin{aligned}
\rho_{I-A}= & \frac{3}{8 \pi L_{P}^{2}} H_{I-A}^{2} \\
= & \frac{3}{8 \pi L_{P}^{2}}\left[\frac{313 \pi^{14} k^{14} c^{14} L_{P}^{2}}{T_{D 3}^{8} \sigma_{0}^{73}}\right. \\
\times & {\left[1-\left[1-\frac{\tau^{2}}{\tau_{0}^{2}}\right]^{1 / 2} \frac{T_{0}^{2}}{T^{2}}\right]^{8}\left[1-\left[1-\frac{\sigma^{2}}{\sigma_{0}^{2}}\right]^{1 / 2} \frac{T_{0}^{2}}{T^{2}}\right]^{5} } \\
\times & {\left[1-\left[1-\frac{\tau^{2}}{\tau_{0}^{2}}\right]^{1 / 2} \frac{T_{0}^{2}}{T^{2}}\right]^{73} } \\
\times & {\left.\left[1+\frac{8 \pi^{5} k^{5} c^{5} L_{P}^{2}}{T_{D 3}^{313} \sigma_{0}^{313}}\left[1-\left[1-\frac{\sigma^{2}}{\sigma_{0}^{2}}\right]^{1 / 2} \frac{T_{0}^{2}}{T^{2}}\right]^{313}\right]\right]^{2} }
\end{aligned}
$$




$$
\begin{aligned}
& \rho_{I-B}=\frac{3}{8 \pi L_{P}^{2}} \\
& H_{I-B}^{2}=\frac{3}{8 \pi L_{P}^{2}} \times\left[\frac{313 \pi^{14} k^{14} c^{14} L_{P}^{2}}{T_{D 3}^{8} \sigma_{0}^{73}}\right. \\
& \times e^{-\left[1-\left[1-\frac{\tau^{2}}{\tau_{0}^{2}}\right]^{1 / 2} \frac{T_{0}^{2}}{T^{2}}\right]^{8}\left[1-\left[1-\frac{\sigma^{2}}{\sigma_{0}^{2}}\right]^{1 / 2} \frac{T_{0}^{2}}{T^{2}}\right]^{5}} \\
& \times \frac{1}{\left[1-\left[1-\frac{\tau^{2}}{\tau_{0}^{2}}\right]^{1 / 2} \frac{T_{0}^{2}}{T^{2}}\right]^{73}} \\
& \left.\times \frac{1}{\left[1+\frac{8 \pi^{5} k^{5} c^{5} L_{P}^{2}}{T_{D 3}^{313} \sigma_{0}^{313}}\left[1-\left[1-\frac{\sigma^{2}}{\sigma_{0}^{2}}\right]^{1 / 2} \frac{T_{0}^{2}}{T^{2}}\right]^{313}\right]}\right]^{2}
\end{aligned}
$$

The above results show that by increasing the temperature of the BIon and the acceleration of the system, the energy density of the universe which lives in part A of the BIon in region I decreases, while the energy density of the universe in part B of the BIon in region I increases. This means that by increasing the temperature of the system, the energy of one part of an accelerating BIon is going out and entering into another part.

\section{Summary and discussion}

We constructed the Padmanabhan idea in an accelerating BIon, and argued that the birth and expansion of the universe are controlled by the evolution of the BIon in extra dimensions. We have shown that the acceleration of the BIon leads to the difference between the number of degrees of freedom on the boundary surface of the universe and the number of degrees of freedom in a bulk region. Also, we have shown that by increasing the acceleration of the BIon, the scale factor and energy density of the universe grow.
Acknowledgements The work of Alireza Sepehri has been supported financially by the Research Institute for Astronomy and Astrophysics of Maragha (RIAAM), Iran under the Research Project No. 1/5237-79.

The authors would like to sincerely thank an anonymous referee for constructive criticism which led to an improvement in the manuscript.

Open Access This article is distributed under the terms of the Creative Commons Attribution 4.0 International License (http://creativecomm ons.org/licenses/by/4.0/), which permits unrestricted use, distribution, and reproduction in any medium, provided you give appropriate credit to the original author(s) and the source, provide a link to the Creative Commons license, and indicate if changes were made.

Funded by SCOAP ${ }^{3}$.

\section{References}

1. T. Padmanabhan, arXiv:1206.4916 [hep-th]

2. Y.-X.L. KeYang, Y.-Q. Wang, Phys. Rev. D 86, 104013 (2012)

3. Y. Ling, W.-J. Pan, Phys. Rev. D 88, 043518 (2013)

4. A. Sheykhi, Phys. Rev. D 87, 061501(R) (2013)

5. M. Eune, W. Kim, Phys. Rev. D 88, 067303 (2013)

6. D.L. EeChang-Young, J. High Energy Phys. 1404, 125 (2014)

7. A.F. Ali, Phys. Lett. B 732, 335 (2014)

8. A. Sepehri, Phys. Lett. B 741, 9296 (2015)

9. G. Grignani, T. Harmark, C. Kift, A. Marini, M. Orselli, Phys. Rev. D 96, 106023 (2017)

10. T. Harmark, JHEP 07, 043 (2000). arXiv:hep-th/0006023

11. P.M. Alsing, I. Fuentes-Schuller, R.B. Mann, T.E. Tessier, Phys. Rev. A 74, 032326 (2006)

12. H. Ghaforyan, S. Shoorvazi, A. Sepehri, M. Ebrahimzadeh, Eur. Phys. J. C 78, 672 (2018)

13. A. Montakhab, M. Ghodrat, M. Barati, Statistical thermodynamics of a two-dimensional relativistic gas. Phys. Rev. E 79, 031124 (2009)

14. M. Ghodrat, A. Montakhab, Phys. Rev. E 82, 011110 (2010)

15. C. Faras, V.A. Pinto, P.S. Moya, Scientific Reports, "What is the temperature of a moving body?", volume 7, Article number: 17657 (2017). https://doi.org/10.1038/s41598-017-17526-4 